\title{
Long-term survival of cemented versus uncemented total hip arthroplasty in the treatment of acute femoral neck fracture: A retrospective observational study with a mean 10-year follow-up
}

\author{
Xiulan Han \\ The First Affiliated Hospital, Sun Yat-sen University \\ Weiguang Yu \\ The First Affiliated Hospital, Sun Yat-sen University \\ Jinluan Lin \\ The Affiliated hospital of Fujian Medical University \\ Mingdong Zhao \\ Jinshan Hospital, Fudan University \\ Guowei Han \\ The First Affiliated Hospital, Sun Yat-sen University \\ Chao Xin Zhang ( $\square$ zhangxc401@aliyun.com ) \\ Jinshan Hospital, Fudan University
}

Research article

Keywords: Total hip arthroplasty, Cemented, Uncemented, Harris Hip Score, Survival

Posted Date: February 27th, 2020

DOI: https://doi.org/10.21203/rs.2.24764/v1

License: (c) (1) This work is licensed under a Creative Commons Attribution 4.0 International License.

Read Full License 


\section{Abstract}

Background Despite the increased use of uncemented total hip arthroplasty (UTHA), there is little evidence of its superiority over cemented total hip arthroplasty (CTHA). The purpose of this retrospective study was to compare the long-term survivorship and Harris Hip Scores (HHSs) of CTHA versus UTHA in the treatment of acute femoral neck fractures (FNFs). Methods Data involving 224 hips (CTHA, $n=112$; UTHA, $n=112$ ) that underwent primary surgery in our medical institutions during 2005-2017 were analysed retrospectively. The primary endpoint was the risk of all-cause revision. The difference in the risk of all-cause revision between groups was assessed by Kaplan-Meier survival analysis with a log-rank test and Cox regression analysis. Results The mean follow-up from surgery was 10 years (range, 3 - 13 years). Kaplan-Meier estimated that the 10-year implant survival was $98.1 \%$ (Cl: $96.1-98.5)$ in the CTHA group and $96.2 \%(\mathrm{Cl}$ : 95.2-97.3) in the UTHA group $(\mathrm{p}=0.030)$. The adjusted Cox regression analysis demonstrated a lower risk of revision in CTHA than in UTHA (hazard ratio [HR] $=1.4,95 \%$ confidence interval $[\mathrm{Cl}]=1.1-2.6, \mathrm{p}=0.000$ ). At the final follow-up, significant differences were detected in $\mathrm{HHS}$ (85.10[ \pm 12.21$]$ for CTHA vs. 79.11[ \pm 13.19$]$ for UTHA). Conclusion This retrospective analysis demonstrates that CTHA has superior survival to UTHA, with a significantly reduced revision risk and higher functional outcome scores. Further follow-up is necessary to verify whether the CTHA advantage persists over time.

\section{Background}

Femoral neck fractures (FNFs) are severe injuries and may lead to long-term disability[1, 2]. For displaced fractures, cemented or uncemented total hip arthroplasty (CTHA or UTHA, respectively) is frequently used[3]. Implant failure secondary to primary THA has become a concern and is eventually addressed using THA revision, contributing to a revision rate as high as 15 to $35 \%[2,4]$. Nevertheless, there is a paucity of literature on the long-term survivorship and Harris Hip Scores (HHSs) following primary CTHA or UTHA in the Asian population[5].

Although previous literature has reported implant survival and HHSs after primary CTHA or UTHA, they have either focused on complication rates or primary diagnoses[6, 7]. Additionally, the reported results for implant survival and HHSs vary widely and are mainly attributable to differences in inclusion and exclusion criteria, sample sizes, and the length of follow-up[8, 9]. Data on long-term implant survival and HHSs remain lacking. Furthermore, limited literature has reported implant survival; however, most of these reports have been subject to the length of follow-up[10]. A previous study reported by Lazarinis showed approximately a revision rate of $7.5 \%$ after 10 years for CTHA[11]. More recent studies have almost all focused on the application of UTHA and have shown exceptional results, with 5-year survival rates approaching $90-100 \%[6,12]$. However, these first-rate results have not been verified in other studies, e.g., Swarup reported a 10-year survival rate of $87 \%$ for UTHA[8].

To date, there remains limited evidence for the superiority of CTHA over UTHA in terms of implant survival and HHS. As THA volume continues to increase, understanding the two indexes occurring throughout the 
postoperative period will be especially valuable. Hence, we performed this retrospective study to compare the long-term survivorship and HHS of CTHA versus UTHA in the treatment of acute FNFs in the Asian population.

\section{Methods}

\section{Study population}

Between January 2005 and August 2017, data involving patients who underwent unilateral primary CTHA or UTHA for acute FNFs were collected from our tertiary academic medical centre. Manufacturer details of the stems and cups employed in THA are shown in Table 1. The main exclusion criteria included inadequate study-related data, secondary CTHA or UTHA, bed-ridden status prior to primary CTHA or UTHA, pathological fracture secondary to malignant diseases, incapacity to follow instructions, osteoarthritis or rheumatoid arthritis, active hip infection, tumour, injury-severity score (ISS) $>10$, multiple injuries, severe cognitive impairment, drug or alcohol abuse, and an American Society of Anesthesiologists (ASA) score of IV or V.

Based on our criteria, a total of 112 patients (112 hips) with complete data were identified from our medical centre and were included in the survival analysis, as shown in Figure 1. All surgical procedures were carried out at our medical centre by 3 high-volume orthopaedists ( $W Y, X Z, M Z$ ), all of whom were experienced with arthroplasties. The indications for surgery included FNFs (OTA/AO, 31-B1, 2, 3). A consistent protocol of antibiotics (cefazolin, $1.0 \mathrm{~g}$; ivgtt, bid, Baomanbio, Shanghai, China) was used perioperatively for 3 days. Furthermore, an anticoagulant regimen (enoxaparin sodium, Clexane 4000 AXa IU, ivgtt; qd, AVENTIS, Shanghai, China) was used for each patient without contraindications for 1 month, starting at admission. A similar rehabilitation programme was used for each patient. Early mobilisation was advocated, with weight bearing as tolerated. The follow-up data included the risk of all-cause revision and the HHS. Follow-up occurred 1 year, 2 years, and every 2 years after primary CTHA or UTHA. The primary endpoint was implant survival.

\section{Statistical analysis}

Implant survival was calculated from the initiation of primary CTHA or UTHA until the date of final followup or revision surgery, whichever occurred first. Signs of cemented or uncemented stem loosening were evaluated by 2 co-authors in accordance with previous reports[13, 14], and the assessment of the cups was performed using Charnley zones[15]. Revision was defined as the removal or exchange of any component[7]. Failure was defined as revision of the primary CTHA or UTHA for any cause. The difference in risk of revision between groups was assessed by Kaplan-Meier survival analysis with a logrank test and Cox regression analysis. A Cox proportional hazard model was utilised to estimate the hazard ratio (HR) and corresponding 95\% confidence interval (Cl) for survival. The Kaplan-Meier method and Cox analysis were performed with adjustments for age, sex, body mass index (BMI), bone mineral density (BMD), and ASA score. Categorical variables were compared using Pearson's $\chi^{2}$ test or Fisher's 
test, as appropriate. Statistical analyses were performed using SPSS (version 24.0, IBM Corp, Armonk, NY, USA). The level of significance was set at $p=0.05$.

\section{Results}

\section{Overall survivorship}

For the survival analysis, cases with at least a 7-year follow-up were included. There were $102(45.1 \%)$ males and 124 (54.9\%) females, with a median age of 68.8 (60-77) years. At the time of analysis, the median follow-up was 10.2 (7-13) years. The median interval to THA after FNF was 8.4 (1-15) days. No statistically significant differences were detected regarding baseline characteristics between groups. $A$ study flow chart is illustrated in Figure 1, and the baseline data are demonstrated in Table 2.

The results of the survival analysis are shown in Figure 2. CTHA had a significantly better 10-year survival of $98.1 \%$ (Cl: $96.1-98.5)$ than that estimated at 10 years for UTHA. UTHA had an estimated 10-year survival of $96.2 \%$ (Cl: 95.2-97.3). Statistically significant differences existed in the 10-year survival for CTHA versus UTHA $(p=0.030)$. Cox regression demonstrated similar results; patients treated with a UTHA had a 1.4-fold increase in risk of revision after adjusting for age, sex, BMI, BMD, and ASA score.

\section{Functional outcomes}

The mean HHS increased from $55.74( \pm 10.32)$ prior to surgery to $85.10( \pm 12.21)$ at the final follow-up for CTHA patients and $56.16( \pm 11.24)$ to $79.11( \pm 13.19)$ for UTHA patients. Approximately $72 \%$ of the patients who were treated with CTHA or UTHA had a satisfactory HHS at the final follow-up. Table 3 presents the values for each follow-up after surgery. The mean HHSs in the CTHA and UTHA groups were $85.12( \pm 8.22)$ and $84.73( \pm 6.21)$ one year after surgery, respectively $(p=0.104)$. From 2 years after surgery to the final follow-up, CTHA yielded higher HHSs than UTHA (all $p<0.05$ ). Especially at the final follow-up, CTHA patients had a significantly higher HHS of $85.10( \pm 12.21)$ compared to UTHA patients, who had an HHS of $78.11( \pm 13.19)(p=0.000)$.

\section{Discussion}

This retrospective study provides evidence that CTHA has superior survival to UTHA, with the revision risk being significantly decreased and the HHS being higher. To our knowledge, this is a relatively large sample size study involving survivorship between CTHA and UTHA following acute FNFs.

Several limitations exist in the present study. First, the retrospective nature of our study tends to lead to errors in evaluating patient characteristics and could be associated with limited power to draw robust conclusions. Second, selection bias exists objectively and is difficult to avoid because of the exclusion of a large number of undesirable cases. Third, follow-up outcomes are likely to be influenced by patient-level confounders and the experience of the orthopaedists. Nonetheless, abiding by arthroplasty guidelines 
and having a high-volume environment ensure that we optimise the treatment of patients. Despite the drawbacks mentioned above, the error margin seems to be acceptable in the present setting.

A growing but still extremely limited body of literature assessing survivorship between CTHA and UTHA showed a survival advantage for CTHA[16]. In the current report, we observed a reduced survival for UTHA, with a 10 -year survival approaching $96.2 \%$. However, a 10 -year survival of $57 \%$ was reported in a previous study involving 10 cases treated with secondary UTHA[7]. In addition, a 10-year survival of 77\% occurred in 20 UTHA cases[17]. The phenomena of low 10-year survival for UTHA was possibly triggered by poor patient survival data and a small sample size. Junnila et al.[18] reported a brand-level comparison of CTHA based on the Nordic Arthroplasty Register Association (NARA) database and showed that implant survival was above the acceptable limit for 10-year survival (95.8\%). Laaksonen et al.[19] reported 10,113 primary UTHAs and demonstrated that the overall survivorship up to 8 years was 94.4-96.25\%. However, a recent study showed no significant difference in survival between CTHA and UTHA[10]. This may partially be attributed to the low numbers (22 hip) in this study. Recently, 10-year survival after THAs has been reported in with large samples (i.e., more than 100 hips). Davis et al.[20] reported a 10-year survival of $95 \%$ in a series of 104 CTHA-treated hips. Additionally, a 10-year survival of $97 \%$ in a series of 112 CTHA-treated hips was noted in a study by Norambuena et al.[21]. In Europe, the 10-year survival for CTHA was higher than that for UTHA[22, 23]. The superiority of hip stability after CTHA over that after UTHA has been reported due to the low stress transfer and good fusion of the cement-bone interface[7, 24]. The between-component mechanical wear introduced by edge loading is lower in CTHA than in UTHA[24]. Along with our findings, the results reported by these studies seem to confirm that CTHA is the preferred alternative for managing an acute FNF.

This study showed that CTHA had a lower revision risk than UTHA. Revision risks for CTHA reported in earlier studies tend to be miscellaneous, with revision risks ranging from $3.2 \%$ to $11 \%[24,12,25]$. Despite the low revision risk for CTHA in the present study, when previous reports assessing revision risks in CTHA are regarded as a reference, the revision risk is consistent with previously reported values[25]. Pedersen et al.[4], who used the Nordic Arthroplasty Registry Association database, found that UTHA rather than CTHA in patients younger than 55 years had more short-term revisions owing to dislocation, periprosthetic fracture and infection. Recently, several reports assessing CTHA have shown a lower revision risk at the midterm follow-up compared with those assessing UTHA[26, 27]. UTHA appears to be associated with an increased risk of revision following primary THA[28].

\section{Conclusions}

The long-term results reported in this study support an increasing body of evidence that CTHA is associated with increased survival benefits, lower revision risk, and higher HHSs compared with UTHA. Hence, when proceeding with UTHA in patients with an acute FNF, the patients should be informed of the increased potential for failure, revision, and poor HHS. Furthermore, when interpreting the differences in survival benefit or revision risk for CTHA and UTHA, strategies to improve implant survival should be directed based on baseline characteristics, such as age, sex, BMI, BMD, and ASA score. 


\section{Abbreviations}

CTHA: cemented total hip arthroplasty; UTHA: uncemented total hip arthroplasty; FNF: femoral neck fracture; HR: hazard ratio; $\mathrm{Cl}$ : confidence interval; HHS: harris hip score; BMI: body mass index; BMD: bone mineral density; ISS: injury severity score; ASA: American Society of Anesthesiologists; SD: Standard deviation.

\section{Declarations}

\section{Acknowledgements}

The authors would like to thank Hang Yu for her help with retrieval of data.

\section{Funding}

Funding for this research was received from the National Natural Science Foundation of China (Grant No. 81971315).

\section{Availability of data and materials}

The datasets used and/or analysed during the current study are available from the corresponding author upon reasonable request.

\section{Authors' contributions}

$\mathrm{XH}$ performed the data collection and analysis and participated in manuscript writing. WY and JL performed the database setup and statistical analysis. MZ and GH performed the operations. XZ participated in the study design and coordination and helped to draft the manuscript. All authors have read and approved the final manuscript.

\section{Ethics approval and consent to participate}

This study was approved by the Medical Ethics Committee (The First Affiliated Hospital, Sun Yat-sen University), and an exemption from informed consent was obtained from our responsible Investigational Ethics Review Board.

\section{Consent for publication}

Not applicable.

\section{Competing interests}

The authors declare that they have no competing interests.

\section{References}


1. Zijlstra WP, De Hartog B, Van Steenbergen LN et al. Effect of femoral head size and surgical approach on risk of revision for dislocation after total hip arthroplasty An analysis of 166,231 procedures in the Dutch Arthroplasty Register (LROI). Acta Orthop. 2017;88(4):395-401. 2. Parry JA, Hernandez NM, Berry DJ et al. Risk Factors for Subsidence of Modular Fluted Tapered Stems Used During Revision Total Hip Arthroplasty for Periprosthetic Hip Fractures. J Arthroplasty. 2018;33(9):2967-70. 3. Abdel MP, von Roth P, Harmsen WS et al. What is the lifetime risk of revision for patients undergoing total hip arthroplasty? A 40-YEAR OBSERVATIONAL STUDY OF PATIENTS TREATED WITH THE CHARNLEY CEMENTED TOTAL HIP ARTHROPLASTY. Bone Joint J. 2016;98B(11):1436-40. 4. Pedersen AB, Mehnert F, Havelin LI et al. Association between fixation technique and revision risk in total hip arthroplasty patients younger than 55 years of age. Results from the Nordic Arthroplasty Register Association. Osteoarthritis Cartilage. 2014;22(5):659-67. 5. Cloke DJ, Khatri M, Pinder IM et al. 284 press-fit Kinemax total knee arthroplasties followed for 10 years - Poor survival of uncemented prostheses. Acta Orthop. 2008;79(1):28-33. 6. Swarup I, Marshall AC, Lee YY et al. Implant survival and patient-reported outcomes after total hip arthroplasty in young patients with developmental dysplasia of the hip. Hip Int. 2016;26(4):367-73. 7. Clarke-Jenssen J, Westberg M, Roise 0 et al. Reduced survival for uncemented compared to cemented total hip arthroplasty after operatively treated acetabular fractures. Injury. 2017;48(11):2534-9. 8. Swarup I, Lee YY, Chiu YF et al. Implant Survival and Patient-Reported Outcomes After Total Hip Arthroplasty in Young Patients. J Arthroplasty. 2018;33(9):2893-8. 9. Swarup I, Lee YY, Christoph El et al. Implant Survival and Patient-Reported Outcomes After Total Hip Arthroplasty in Young Patients With Juvenile Idiopathic Arthritis. J Arthroplasty. 2015;30(3):398-402. 10. Lamb JN, Matharu GS, Redmond A et al. Patient and implant survival following intraoperative periprosthetic femoral fractures during primary total hip arthroplasty AN ANALYSIS FROM THE NATIONAL JOINT REGISTRY FOR ENGLAND, WALES, NORTHERN IRELAND AND THE ISLE OF MAN. Bone Joint J. 2019;101B(10):1199-208. 11. Lazarinis S, Karrholm J, Hailer NP. Increased risk of revision of acetabular cups coated with hydroxyapatite A Swedish Hip Arthroplasty Register study involving 8,043 total hip replacements. Acta Orthop. 2010;81(1):53-9. 12. Thillemann TM, Pedersen AB, Mehnert F et al. The Risk of Revision After Primary Total Hip Arthroplasty Among Statin Users A Nationwide Population-Based Nested Case-Control Study. J Bone Joint Surg Am. 2010;92A(5):1063-72. 13. Gruen TA, McNeice GM, Amstutz HC. "Modes of failure" of cemented stem-type femoral components: a radiographic analysis of loosening. Clin Orthop Relat Res. 1979(141):17-27. 14. Engh CA, Massin P, Suthers KE. Roentgenographic assessment of the biologic fixation of porous-surfaced femoral components. Clin Orthop Relat Res. 1990(257):107-28. 15. DeLee JG, Charnley J. Radiological demarcation of cemented sockets in total hip replacement. Clin Orthop Relat Res. 1976(121):20-32. 16. Faris PM, Ritter MA, Keating EM et al. The cemented all-polyethylene acetabular cup: Factors affecting survival with emphasis on the integrated polyethylene spacer - An analysis of the effect of cement spacers, cement mantle thickness, and acetabular angle on the survival of total hip arthroplasty. $J$ Arthroplasty. 2006;21(2):191-8. 17. Howard JL, Kremers HM, Loechler YA et al. Comparative Survival of Uncemented Acetabular Components Following Primary Total Hip Arthroplasty. J Bone Joint Surg Am. 2011;93A(17):1597-604. 18. Junnila M, Laaksonen I, Eskelinen A et al. Implant survival of the most common cemented total hip devices from the Nordic Arthroplasty Register Association database. Acta Orthop. 2016;87(6):546-53. 19. Laaksonen I, Lorimer M, Gromov K et al. Trabecular metal acetabular 
components in primary total hip arthroplasty Higher risk for revision compared with other uncemented cup designs in a collaborative register study including 93,709 hips. Acta Orthop. 2018;89(3):259-64. 20. Davis ET, Pagkalos J, Kopjar B. Polyethylene manufacturing characteristics have a major effect on the risk of revision surgery in cementless and hybrid total hip arthroplasties AN ANALYSIS OF THE NATIONAL JOINT REGISTRY OF ENGLAND, WALES, NORTHERN IRELAND AND THE ISLE OF MAN. Bone Joint J. 2020;102B(1):90-101. 21. Norambuena GA, Wyles CC, Van Demark RE et al. Effect of dislocation timing following primary total hip arthroplasty on the risk of redislocation and revision. Hip Int. 2019;29(5):48995. 22. Kuijpers MFL, Hannink G, Vehmeijer SBW et al. The risk of revision after total hip arthroplasty in young patients depends on surgical approach, femoral head size and bearing type; an analysis of 19,682 operations in the Dutch arthroplasty register. BMC Musculoskelet Disord. 2019; 20(1):385. 23. Bryan AJ, Calkins TE, Karas V et al. Primary Total Hip Arthroplasty in Patients Less Than 50 Years of Age at a Mean of 16 Years: Highly Crosslinked Polyethylene Significantly Reduces the Risk of Revision. J Arthroplasty. 2019;34(7):S238-S41. 24. Engesaeter LB, Espehaug B, Lie SA et al. Does cement increase the risk of infection in primary total hip arthroplasty? Revision rates in 56,275 cemented and uncemented primary THAs followed for 0-16 years in the Norwegian Arthroplasty Register. Acta Orthop. 2006;77(3):351-8. 25. Hailer NP, Weiss RJ, Stark A et al. The risk of revision due to dislocation after total hip arthroplasty depends on surgical approach, femoral head size, sex, and primary diagnosis An analysis of 78,098 operations in the Swedish Hip Arthroplasty Register. Acta Orthop. 2012;83(5):442-8. 26. Paxton E, Cafri G, Havelin L et al. Risk of Revision Following Total Hip Arthroplasty: Metal-on-Conventional Polyethylene Compared with Metal-on-Highly Cross-Linked Polyethylene Bearing Surfaces. J Bone Joint Surg Am. 2014;96A:19-24. 27. Varnum C, Pedersen AB, Kjaersgaard-Andersen P et al. Comparison of the risk of revision in cementless total hip arthroplasty with ceramic-on-ceramic and metal-on-polyethylene bearings Data on 11,096 patients from the Danish Hip Arthroplasty Registry. Acta Orthop. 2015;86(4):477-84. 28. Varnum $\mathrm{C}$, Pedersen AB, Makela $\mathrm{K}$ et al. Increased risk of revision of cementless stemmed total hip arthroplasty with metal-on-metal bearings Data from the Nordic Arthroplasty Register Association. Acta Orthop. 2015;86(4):469-76.

\section{Tables}

Table 1 Manufacturer details of stems and cups employed in THA

\begin{tabular}{|c|c|c|}
\hline Patients, No. & Stem & Cup \\
\hline \multicolumn{3}{|l|}{ CTHA } \\
\hline 54 & Exeter ${ }^{1}$, & Exeter $^{1}$, Elite $^{4}, \mathrm{IP} / \mathrm{SP} 1^{3}$ \\
\hline 32 & Spectron $\mathrm{EF}^{2}$, & Contemporary $^{1}$, Marathon ${ }^{4}$, \\
\hline 26 & Lubinus SP2 ${ }^{3}$, & Exeter $\mathrm{X}^{3}$ Rimfit $^{1}$ \\
\hline \multicolumn{3}{|l|}{ UTHA } \\
\hline 75 & Corail $^{4}$, & Reflection uncemented $^{2}$, \\
\hline 39 & Filler ${ }^{5}$ & Trident $^{1}$, Igloo $^{5}$ \\
\hline
\end{tabular}


Table 2 Patient demographics and outcomes

\begin{tabular}{|c|c|c|c|}
\hline Variable & CTHA $(n=112)$ & UTHA $(n=114)$ & $p$-value \\
\hline Sex, No. M/F & $52 / 60$ & $50 / 64$ & $0.698^{\mathrm{a}}$ \\
\hline Age at surgery(years) & $68.75 \pm 8.36$ & $69.02 \pm 8.22$ & $0.171^{\mathrm{b}}$ \\
\hline $\mathrm{BMI} \mathrm{kg} / \mathrm{m}^{2}$ & $25.62 \pm 8.34$ & $26.14 \pm 7.53$ & $0.106^{\mathrm{b}}$ \\
\hline $\mathrm{BMD}$ & $-2.74 \pm 0.65$ & $-2.75 \pm 0.43$ & $0.212^{\mathrm{b}}$ \\
\hline Side, No. left/right & $54 / 58$ & $59 / 55$ & $0.595^{\mathrm{a}}$ \\
\hline Comorbidities & & & $0.757^{\mathrm{c}}$ \\
\hline Hypertension & 37 & 40 & \\
\hline Diabetes mellitus & 34 & 28 & \\
\hline Hypertension and Diabetes mellitus & 11 & 14 & \\
\hline Pulmonary & 8 & 10 & \\
\hline Cerebrovascular accident & 9 & 7 & \\
\hline Cardiopathy & 7 & 8 & \\
\hline Anemia & 6 & 7 & \\
\hline Mechanism of injury & & & $0.537^{\mathrm{C}}$ \\
\hline Traffic-related injury & 31 & 27 & \\
\hline Injury by falling & 60 & 64 & \\
\hline Tamp injury & 21 & 23 & \\
\hline ASA Index & & & $0.313^{\mathrm{c}}$ \\
\hline ] & 23 & 32 & \\
\hline [ & 57 & 52 & \\
\hline Q & 32 & 30 & \\
\hline Fracture types, OTA/AO & & & $0.518^{\mathrm{c}}$ \\
\hline 31-B1 & 36 & 31 & \\
\hline 31-B2 & 57 & 63 & \\
\hline 31-B3 & 19 & 20 & \\
\hline Surgical approach & & & $0.696^{\mathrm{c}}$ \\
\hline Anterior & 54 & 52 & \\
\hline Anterolateral & 58 & 62 & \\
\hline Time to surgery (days) & $8.00 \pm 7.32$ & $8.00 \pm 6.75$ & $0.241^{b}$ \\
\hline Head size, $\mathrm{mm}$ & & & $0.962^{\mathrm{c}}$ \\
\hline 28 & 32 & 35 & \\
\hline 30 & 34 & 27 & \\
\hline 32 & 28 & 34 & \\
\hline 36 & 20 & 18 & \\
\hline Preoperative HHS & $55.74 \pm 10.32$ & $56.16 \pm 11.24$ & $0.528^{\mathrm{b}}$ \\
\hline Follow-up time (years) & $10.33 \pm 3.15$ & $10.21 \pm 3.01$ & $0.312^{\mathrm{b}}$ \\
\hline
\end{tabular}

a Analysed using the chi-square test; ${ }^{b}$ Analysed using an Independent-Samples t-test;

${ }^{c}$ Analysed using the Mann-Whitney test. CTHA: cemented total hip arthroplasty; UTHA: uncemented total hip arthroplasty; HHS: Harris hip score; ASA: American Society of Anaesthesiologists; BMI: body mass index; BMD: bone mineral density. 


\begin{tabular}{llll}
\hline HHS, year(s) postoperatively & CTHA $(\mathrm{n}=112)$ & UTHA $(\mathrm{n}=114)$ & $p$-value \\
\hline 1 & $85.12 \pm 8.22$ & $84.73 \pm 6.21$ & $0.104^{*}$ \\
2 & $89.53 \pm 8.42$ & $86.37 \pm 7.26$ & $0.021^{*}$ \\
4 & $86.15 \pm 8.37$ & $84.30 \pm 8.25$ & $0.014^{*}$ \\
6 & $87.62 \pm 7.35$ & $85.16 \pm 9.20$ & $0.012^{*}$ \\
8 & $85.57 \pm 9.26$ & $80.38 \pm 7.67$ & $0.010^{*}$ \\
\hline 10 & $85.48 \pm 11.03$ & $79.61 \pm 12.15$ & $0.010^{*}$ \\
\hline At final follow-up & $85.10 \pm 12.21$ & $78.11 \pm 13.19$ & $0.000^{*}$ \\
\hline
\end{tabular}

*Statistically significant values.

CTHA: cemented total hip arthroplasty; UTHA: uncemented total hip arthroplasty; HHS: Harris hip score.

\section{Figures}

Between January 2005 and August 2017, 298 consecutive patients who underwent a unilateral primary CTHA or UTHA for acute FNFs were identified

Reasons for exclusion $(n=72)$

-inadequate study-related data $(n=16)$

-secondary CTHA or UTHA $(n=6)$

-bed-ridden status prior to primary CTHA

or UTHA $(n=6)$

-pathological fracture secondary to

malignant diseases $(n=5)$

-incapacity to follow instructions $(n=8)$

-osteoarthritis or rheumatoid arthritis $(n=$

3)

-active hip infection $(n=2)$

-tumour $(\mathrm{n}=5)$

-an injury-severity score (ISS) $>10(n=4)$

-multiple injuries $(n=5)$

-severe cognitive impairment $(n=3)$

-drug or alcohol abuse $(n=3)$

-an ASA score of IV or V $(n=6)$ 
Flow diagram demonstrating methods for identification and exclusion of study to assess the survivorship of CTHA and UTHA in the treatment of acute femoral neck fracture (FNF).

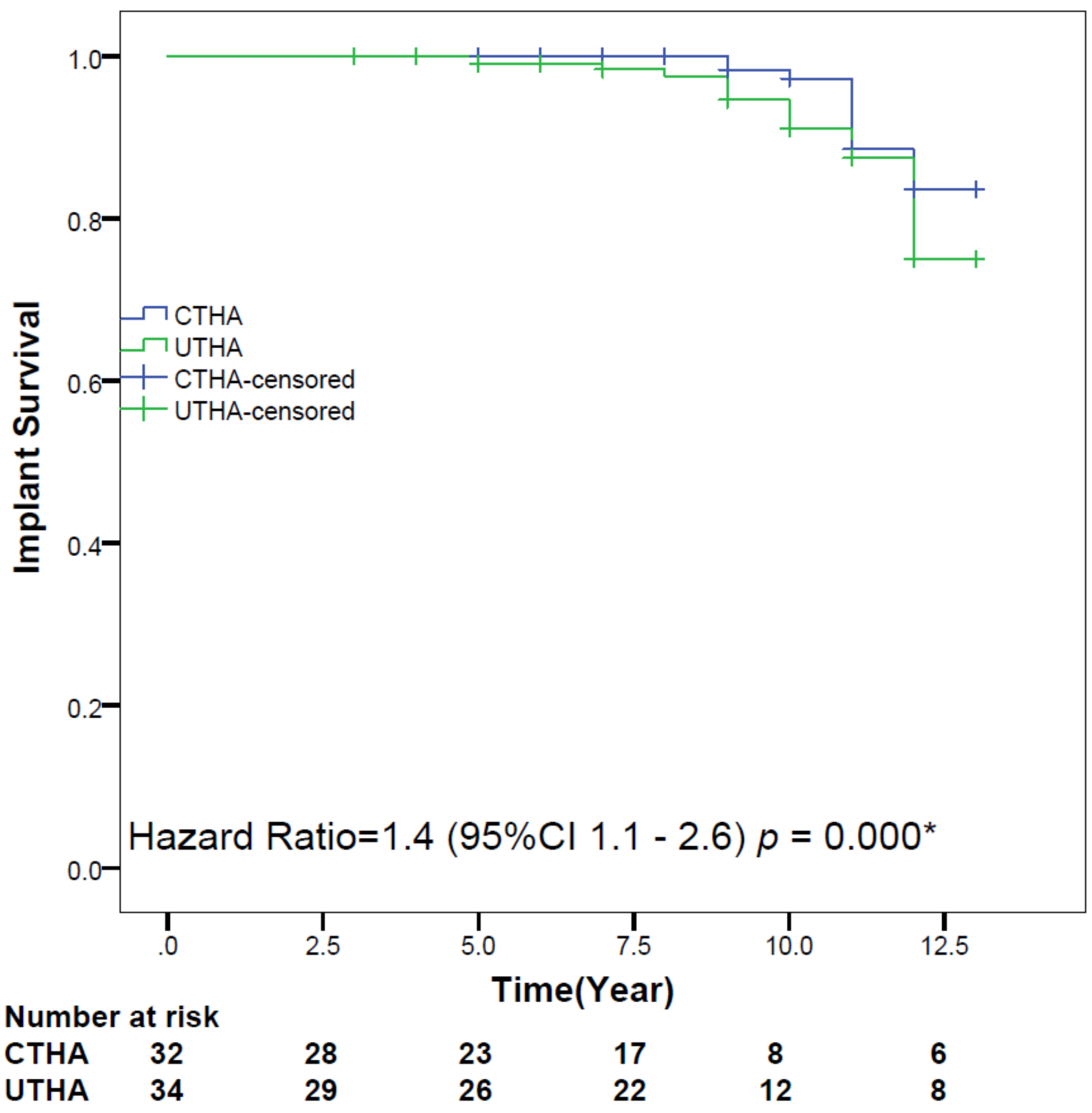

Figure 2

Kaplan-Meier survival analysis comparing CTHA with UTHA using the risk of all-cause revision as the primary endpoint. The cumulative survival rate at 10 years after surgery was observed to be significant between groups $(p=0.030)$. *HR was calculated using the Cox proportional hazards model, with 
adjustment for age, sex, body mass index (BMI), bone mineral density (BMD), and ASA score as covariates and CTHA/UTHA surgery as the time-dependent factor. 